\title{
Özgür ve Açık Kaynak Kodlu Yazılım Platformlarının Uygulamalı Yapay Zeka Ĕgitimlerine Katkısı
}

\author{
H. Selcuk Nogay ${ }^{\mathrm{a} 1, *}$, Tahir Cetin Akınc1 ${ }^{\mathrm{b} 2}$, Serhat Şeker ${ }^{\mathrm{b} 3}$ \\ a Kayseri Üniversitesi, Mustafa Çıkrıkçıŏglu MYO, Elektrik ve Enerji Bölümü, Kayseri, Türkiye. \\ b İstanbul Teknik Üniversitesi, Elektrik Mühendisliği Bölümü, İstanbul, Türkiye.
}

İstanbul Sabahattin Zaim Üniversitesi Fen Bilimleri Enstitüsü Dergisi (2021) 3 (1): 11-14

https://doi.org/10.47769/izufbed.859979

(iD) $O R C I D{ }_{1}^{1} 0000-0001-9105-508 X ;{ }^{2} 0000-0002-4657-6617 ;{ }^{3} 0000-0001-5816-2211$

\begin{tabular}{l}
\hline YAYIN BİLGİSI \\
\hline Yayın geçmişi: \\
Gönderilen tarih: 13 Ocak 2021 \\
Kabul tarihi: 31 Ocak 2021 \\
\hline Anahtar kelimeler: \\
Yapay Zeka \\
Açık Kaynak Kodlu Yazılım \\
Kapalı Kaynak Kodlu Yazılım \\
Yazılım Mühendisliği \\
Eğitim
\end{tabular}

\begin{abstract}
ÖZET
Açık Kaynak Kodlu (AKK) yazılım ortamları öğrencilere ve araştırmacılara yapay zeka alanında geniş uygulama yapma ve model geliştirme imkanı sunmaktadır. Teknolojinin gelişmesiyle AKK yazılım ortamlarının sayısının artması sonucunda veri bilimi ve veri mühendisliğinde gelişmeler olmuş ve açık kaynak veri tabanları ortaya çıkmıştır. Açık kaynak veri tabanları, bir kapalı kaynak kodlu (KKK) yazılım ortamı olan MATLAB içerisinde kullanılarak yapay zeka alanında yeni gelişmelere yol açmıştır. Bugün tensorflow ve keras gibi açık kaynak yazılım kütüphaneleri sayesinde öğrenciler yapay zeka alanında özgürce tasarım yapma ve geliştirme imkanına sahip olabilmektedir. Bu çalışmada AKK yazılım ortamlarının ve açık kaynak veri tabanlarının yapay zeka eğitimine yaptığı katkılar ele alınmış ve tartışılmıştır. Öğrencilerin üniversite eğitimi süresince AKK yazılım platformlarına erişebilmelerini sağlayacak ve onlara AKK platformunda uygulamalar ve yeni tasarımlar yapma becerisi kazandırabilecek imkanlar sunmak için ilgili bölümlerin müfredat programlarında değişiklikler yapılması gerekliliği ortaya çıkmıştır.
\end{abstract}

\section{Contribution of Free and Open Source Software Platforms to Applied Artificial Intelligence Training}

\begin{tabular}{l}
\hline ARTICLE INFO \\
\hline Article history: \\
Received: 13 January 2021 \\
Accepted: 31 January 2021 \\
\hline Key words: \\
Artificial Intelligence \\
Opoen Source Software \\
Closed Source Software \\
Software Engineering \\
Education
\end{tabular}

\begin{abstract}
Open source software (OSS) environments offer students and researchers the opportunity to develop a wide range of applications and models in the field of artificial intelligence. As a result of the increase in the number of OSS software environments with the development of technology, there have been developments in data science and data engineering and open source databases have emerged. Open source databases have been used in MATLAB, a closed source software (CSS) environment, leading to new developments in the field of artificial intelligence. Today, thanks to open source software libraries such as tensorflow and keras, students can freely design and develop in the field of artificial intelligence. In this study, the contributions of OSS environments and open source databases to artificial intelligence education were discussed and discussed. The necessity of making changes in the curriculum of the relevant departments has emerged in order to enable students to access OSS platforms during their university education and to provide them with the ability to make applications and new designs on the OSS platform.
\end{abstract}

\section{Giris}

AKK yazılımlar, tüm kurum ve bireylerin rahatça ulaşabileceği, kullanabileceği, kendi gereksinimlerine göre geliştirip uyarlayabileceği yazılımlardır. AKK yazılımlar, her alanda birçok problem için çözümler sunarak, yazılım tekellerine karşı tüketiciye geniş çapta seçenekler ve önemli uygulama ve geliştirme firsatları sunmaktadır. Bazı popüler açık kaynak yazılım ürünleri sürekli geliştirmeye tabi tutulur ve ticari benzerlerinden daha güvenilir olma eğilimindedir (Dorodchi ve Dehbozorgi 2016).

AKK yazılım ortamları teknoloji üretebilecek ve ülke ekonomisine büyük katkı sağlayabilecek gençleri yetiştirmenin etkin alternatif yollarından birisidir. Tüm sektörlerde bilişim maliyetinin gittikçe arttığı bilinen bir gerçektir. AKK yazılımlar, bu maliyetleri düşürerek, yerli 
sanayici ve bilişimciye destek olarak ülkenin rekabet yeteneğinin artmasına yardımcı olabilir. Günümüzde tüm dünyada AKK yazılım platformları ve açık kaynak veri tabanları sayesinde AKK yazılımları, KKK yazılımlarla yetenek ve performans açısından yarışabilecek düzeye gelmiştir. Halen gelişmekte olan ve giderek popülaritesini arttırmakta olan bu AKK yazılım platformları sayesinde gençlerin ülke ekonomisine katkı sağlayabilecekleri düzeye gelebilmelerinin ilk adımı eğitim öğretim kurumlarından geçmektedir. AKK yazılım platformları yazılım mühendisliği, bilişim, veri mühendisliği veri bilimi ve bilgisayar bilimi dallarında öğrenim görmekte olan öğrencilere hitap eden uygulama alanlarını kapsamaktadır. Orta öğretimin lise seviyesinden başlayarak üniversite eğitimine kadar öğrenciler bu uygulama alanlarında tecrübe kazanarak AKK platformlarını kendi ilgi alanları doğrultusunda kullanabilirler ve en popüler alanlarda kendilerini geliştirebilirler. Son yıllarda yazılım mühendislerinin ve ilgili meslek alanlarındaki öğrenci, eğitmen ve araştırmacı olarak faaliyet gösteren herkesin ilgisini çeken alan yapay zekadır. Üniversitelerin lisans, yüksek lisans ve doktora düzeyinde yapılan eğitim öğretim faaliyetlerinde yapay zeka ile ilgili derslerin uygulamalı olarak gerçekleştirilmesi etkin bir eğitim öğretim anlayışı açısından oldukça büyük önem arz etmektedir. Mesleki ve teknik eğitimin her alanında olduğu gibi yapay zeka yöntemlerinin de pratik uygulamalar eşliğinde öğrenilmesi gerekir.

$\mathrm{Bu}$ çalışmada, üniversitelerde öğrencilere ücretsiz olarak kullanabilecekleri ve pratik uygulamalar gerçekleştirebilecekleri AKK yazılım platformlarının yapay zeka eğitimine yapabileceği katkılar araştırılmış yapay zeka eğitim ortamlarının AKK platformlarına dayalı olarak güçlendirilmesi için çözüm önerileri sunulmuştur.

\section{2. İlgili çalışmalar ve görüşler}

Kapalı kaynak kodlu (KKK) ticari ve paket programları ile yapılan uygulamaların veya verilen eğitimlerin çok büyük avantajlarının olduğu şüphesizdir. Ancak, KKK yazılım platformu ile geniş sınırlar içerisinde uygulama yapabilme imkanına sahip olabilmek için bu tür ticari ürünlerin maliyetlerinin (servis, bakım, lisans vs) üstesinden gelmek gerekmektedir. Ayrıca maliyete ek olarak, KKK yazılım platformları, geliştirme ve tasarım platformlarını sağlayıcının belirlediği sınırlar çerçevesinde kullanıcıya sunmaktadır. Ancak yapay zeka söz konusu olduğunda, en yaygın kullanılan KKK platformu olarak bilinen MATLAB, diğer KKK yazılım platformlarından daha farklı olarak ele alınabilir. Yapay zeka teknolojisinin dünyada tanınmasına ve gelişmesine büyük katkı sunan MATLAB ortamı sayesinde birçok bilimsel çalışmaya bugün literatürde rastlamak mümkündür. MATLAB' in yapay zeka alanında son zamanlarda gösterdiği başarısının asıl kaynağı başkadır. Şöyle ki, yapay zeka uygulamalarının başarısının en önemli sebeplerinden birisi veri bilimi ve veri mühendisliği nin gelişmesidir. AKK yazılım platformlarının gelişmeye başlamasıyla veri mühendisliği ve veri bilimi alanında bariz bir şekilde gelişmeler oldu (Drummond ve Alto 2016). Her ne kadar yapay zeka denilince MATLAB ortamı aklımıza gelse bile, bugün AKK yazılım platformları sayesinde ilerleyen bu açık kaynak veri tabanları kullanılarak MATLAB ortamında yapay zeka modelleri gerçekleştirilebilir hale gelmiştir. Başka bir deyişle aslında KKK platformunda bir yapay zeka modeli tasarlarken AKK platformunda üretilen bir veri tabanından yararlanmak gerekmektedir. Dolayısıyla her şekilde AKK yazılım platformları yapay zeka çerçevesinde hayatımıza girmiş bulunmaktadır. Bugün bu veri tabanlarından faydalanarak MATLAB ortamında, tıp alanında akciğer kanseri seviye (Nogay 2017) ve tiplerinin (Nogay vd., 2018a) tespitinde, baş boyun kanseri seviye (Nogay vd., 2018b) ve hayatta kalma süresi (Nogay 2020) tahmininde, farklı kanser tiplerinin sınıflandırılmasında (Nogay 2018), akciğerdeki skuamöz hücreli karsinom yerinin tespitinde (Nogay ve Akıncı 2018) ve otizm erken teşhis çalışmalarında (Nogay ve Adeli 2020), daha birçok bilimsel çalışmada başarı ile uygulandığ1 görülmektedir. MATLAB ortamı makine öğrenme yöntemleri için sunduğu geniş uygulama alanlarını yazılım ile ilgilenen veya ilgilenmeyen her öğrencinin kullanabileceği kolaylık seviyesine getirmeyi başarmıştır. Bununla birlikte AKK yazılım platformlarında tasarlanan modelleri MATLAB ortamına aktararak uygulayabileceği firsatlar sunabilmektedir. MATLAB ortamında gerçekleştirilen makine öğrenme modelleri ile teknikte, elektrik ark kaynak makinelerinin optimum çalışma bölgelerinin tespitinde (Nogay ve Akınc1 2020; Akinci vd., 2011), yenilenebilir enerji kaynaklarında rüzgar enerjisi potansiyeli (Ersoz vd., 2013) ve hız [(Nogay ve Akinci 2012; Nogay vd., 2012a; Nogay ve Akınc1 2019; Akınc1 ve Nogay 2012; Seker vd., 2013; Akınc1 vd., 2016) tahmini çalışmalarında, bina yapı malzemelerinde hasar tespitinde (Volkovas et al 2012), elektrik motorlarında (firçasız doğru akım motoru) hız (Serteller vd., 2012) ve yapısal büyüklükler (Nogay vd., 2012b; Nogay 2016a; Birbir ve Nogay 2007; Birbir vd., 2007; Nogay 2008), iç sıcaklık (Nogay 2011) ve arıza (Nogay 2016b) tahmininde başarılı uygulamalar gerçekleştirilmiştir.

AKK yazılım platformları ile yapay zeka (derin öğrenme ve makine öğrenme) yöntemleri, duygu analizi ve görüntü sinıflandırmada (Lynch et al 2020), kod üretmede (Tang vd., 2019), otomatik kişilik tanımada (Suen vd., 2019), hareket yönü algılamada (Yun ve Woo 2020), baz istasyonu önbelleğinde hangi içeriklerin depolanması gerektiğine karar vermede (Thar vd., 2018) tensorflow ve keras gibi AKK kütüphanelerden faydalanarak başarı ile uygulandığı görülmektedir.

AKK yazılım platformlarının yapay zeka amacı dışında teknik ve normal eğitimde, matematik matematik öğretimine yönelik bilgisayar cebiri, çizim, görselleştirme ve diziye yönelik paket programların eğitim amaçlı kullanımında (Kusbeyzi vd., 2011), STEM eğitiminde büyük veri (big data) ile açık kaynak laboratuvar yazılımında (Xie vd., 2016) başarı ile kullanıldığı ve uygulandığı görülmektedir. AKK yazılım platformları, eğitimciler için, sağlık, eğitim, ekoloji, ekonomik kalkınma, afet müdahalesi ve benzerlerinde bilgi işlem tabanlı çözümler, artan açık kaynak yazılım kullanımıyla birlikte, uygulamalı öğrenme için zengin fursatlar sunmaktadır (Hislop ve Ellis 2017). AKK yazılım platformları yazılım mühendisliği öğrencileri için yeni ve özgür kullanılabilecek tasarım ortamları ve firsatlarının ortaya çıkmasına sebep olmuştur. AKK platformları yardımıyla yazılım mühendisliği eğitimleri bazı bilimsel çalışmalarda ele alındı (Hawthorne ve Perry 2005) ve sonraki yıllarda bir çalışmada sonuçlar hem öğrenci gözüyle (Pinto vd., 2019) ve hem de öğretim elemanı gözüyle 
(Pinto vd., 2017) ayrı ayrı değerlendirildi ve AKK platformlarının öğrencilere ve eğitmenlere büyük firsatlar sağladı̆̆ı ortaya çıktı. Açık Kaynaklı Yazılım (AKK) projelerini bir yazılım mühendisliği dersine sokmanın birçok avantajı vardır; öğrencilerin gerçek dünyadaki projelerden iyi kodlama uygulamalarını öğrenmelerine olanak sağlamak ve öğrencilere gerçek bir proje hakkında bir fikir vermek (Hu vd., 2018) bu avantajlardan sayılabilir.

\section{Sonuç ve Öneriler}

Makine öğrenimi alanındaki gelişmeler genel halkın büyük yararına olabilir. Birçok program ve hatta eğitilmiş ağırlık matrisleri, açık kaynaklı yazılım veya ücretsiz olarak indirilebilir. Bununla birlikte, mevcut haliyle, çoğu sonuç çoğu insan için doğrudan erişilebilir değildir. Makine öğrenimi araçlarını her teknik kişiler için ulaşılabilir hale getirmek yapay zeka alanında daha ileriye gitmek açısından çok önemlidir (Hung 2018). Bu perspektiften bakıldığında AKK yazılım ortamlarının yapay zeka çalışmalar içine ne denli gerekli olduğu daha net anlaşılmaktadır.

Üniversitelerde öğrencilerin hayal gücünü geliştirici tasarımlara yönelmeleri ve kendi tasarımlarını defalarca deneyerek veya uygulamalar yaparak tecrübe ederek özgürce geliştirebilecekleri ortamlara sahip olmaları için araştırma grupları kurulmalı, ve öğrencilerin devam etmekte veya başlayacak olan AKK tabanlı projelere dahil edilmeleri ile tecrübe kazanmaları sağlanabilir (Tao ve Nandigam 2006).

Öğrenciler yerel araştırma gruplarında veya bulut tabanlı hesaplarda kod geliştirebilir. Jupyter Notebook, eğitmenlerin veri görselleştirmeleri, veri madenciliği, bilgisayar simülasyonları, makine öğrenimi ve yapay zeka için etkili bir pedagoji uygulamasına yardımcı olan etkileşimli, açık kaynaklı web uygulaması öğrenme aracıdır. Bugün üniversitelerin mühendislik fakültelerinin ilgili bölümlerinde lisansüstü düzeydeki makine öğrenimi dersleri ve makine öğrenimini içeren lisans düzeyinde yapay zeka vb dersler için Jupyter Notebooks rahatlıkla kullanılabilir ve öğrencilere açık kaynak kodlu yazılım platformunda uygulama imkanı sunulabilir (Bhattacharya et al 2018).

$\mathrm{Bu}$ yaklaşım ile, daha etkili bir öğretim stratejisine yönelik aşağıdaki sonuçlar beklenebilir:

1. Jupyter Notebooks, öğrencilerin büyük verilere ve süper bilgisayara kolay erişimini sağlayabilir. Tüm büyük veriler (Terabayt cinsinden) ve gerekli kütüphaneler uzak bir sunucuda barındırılır. Öğrenciler hiçbir şey yüklemeden veya büyük veri indirmeden algoritmalarını kullanabilir ve deneyebilirler. Jupyter Notebook'u yalnızca, uzak sunucuya bağlanabilen ve büyük veriler üzerinde algoritmalar çalıştırabilen web tarayıcılarında çalıştırabilirler.

2. Öğrenciler birinci sınıftan itibaren öğrenmeye başlayabilirler ve sonuçları, şekilleri, tabloyu görebilirler.

3. Öğrenciler makine öğreniminde hem gerekli bilgileri hem de pratik deneyimleri dikkat çekici bir şekilde edinmeye başlarlar, bu da iş gücüne katılmadan önce özgüvenlerini arttırır.

Eğitim kurumlarının açık kaynak kodlu (AKK) yazılımları eğitim öğretim müfredatına yerleştirmeye başlaması ve öğrencilerin açık kaynak materyallerini tanımaya başlaması önemlidir. Eğitim kurumlarında ve üniversitelerde açık kaynak projeleri geliştirilmeli ve öğrenciler arasında ödüllü yarışmalar yapılarak teşvik edilmelidir. Öğrencilerin bir kısmı için bu projeler deneyim demektir. Bazıları için kişisel olarak tatmin edici işler demektir ve diğer bir kısım için ise yüksek kazanç demektir. Üniversitelerin lisans veya önlisans eğitim kurumlarının bu tür firsatları sağlamaya çalışması gerekmektedir. Üniversitelerde ve hatta ortaöğretim kurumlarında açık kaynak projelerine etkili bir şekilde katılacak gerekli beceri ve deneyime sahip öğrenci yetiştirmek amaçlanmalıdır (DeKoenigsberg 2008).

Kaynaklar

Akinci T. C., Nogay, H. S. , Gokmen, G. (2011). Determination of optimum operation cases in electric arc welding machine using neural network", Journal of Mechanical Science and Technology, 25: 1003-1010.

Akinci, T.C., Nogay, H. S., Guseinoviene, E., Dikun, J., Seker, S., "Application of ANN for Short Term Forecasting of Wind Power Density. (2016). Renewable Energy and Innovative Technologies, Smolyan, Bulgaristan, 10 -11 June, c1 : 157163.

Akinci, T. C., Nogay, H.S. (2012). Wind Speed Correlation Between Neighboring Measuring Stations", Arabian Journal for Science and Engineering, 37:1007-1019.

Bhattacharya, S., Czejdo, B., Agrawal, R., Erdemir, E., Gokaraju, B. (2018). Open Source Platforms and Frameworks for Artificial Intelligence and Machine Learning. SoutheastCon, 19-22 April, IEEE St. Petersburg, FL, USA.

Birbir Y. Nogay, H. S. (2007). Application of Artificial Neural Network for Harmonic Estimation in Different Produced Induction Motors. International Journal of Circuits, Systems and Signal Processing, 4: 334-340, 2007.

Birbir Y., Nogay H. S., Ozel Y. (2007). Neural Network Solution to Low Order Odd Current Harmonics in Short Chorded Induction Motors. International Journal of Systems Applications, Engineering \& Development, 1:21-28, 2007.

DeKoenigsberg, G. (2008). How Successful Open Source Projects Work, and How and Why to Introduce Students to the Open Source World. 21st Conference on Software Engineering Education and Training, 14-17 April, Charleston, SC, USA.

Dorodchi, M., Dehbozorgi, N. (2016). Utilizing Open Source Software in Teaching Practicebased Software Engineering Courses. 2016 IEEE Frontiers in Education Conference (FIE), 12-15 Oct. Erie, PA, USA.

Drummond, D. E., Alto, P. (2016). Open sourcing education for Data Engineering and Data Science. IEEE Frontiers in Education Conference (FIE), 12-15 Oct. Erie, PA, USA.

Ersoz S., Akinci T. C., Nogay H. S., Dogan G. (2013). Determination of Wind Energy Potential in KirklareliTurkey", International Journal of Green Energy, 10:103-116.

Hawthorne M., J., Perry, D. E. (2005). Software Engineering Education in the Era of Outsourcing, Distributed Development, and Open Source Software: Challenges and Opportunities. Proceedings. 27th International Conference on Software Engineering ICSE,15-21 May, 2 Saint Louis, MO, USA.

Hislop, G. W., Ellis, H. J. C. (2017). Humanitarian Open Source Software in Computing Education. Computer IEEE, 50(10):98-101.

Hu, Z., Song, Y., Gehringe, E.F. (2018). Open-Source Software in Class: Students' Common Mistakes. IEEE/ACM 40th International Conference on Software Engineering: Software Engineering Education and Training (ICSE-SEET), 25 May-3 June, Gothenburg, Sweden.

Hung, C. K. (2018). Making Machine-Learning Tools Accessible to Language Teachers and Other Non-Techies: t-sne-lab and Rocanr as First Examples. IEEE 8th International Conference 
on Awareness Science and Technology (iCAST), 8-10 Nov. Taichung, Taiwan.

Kusbeyzi, I., Hacinliyan, A., Aybar, OO. (2011). Open source software in teaching mathematics", Procedia Social and Behavioral Sciences, (15): 769-771, 2011.

Lynch, C., O’Leary, C., Smith, G., Bain, R., Kehoe, J., Vakaloudis, A., Linger, R. (2020). A review of open-source machine learning algorithms for twitter text sentiment analysis and image classification. International Joint Conference on Neural Networks (IJCNN), IEEE, 19-24 July, Glasgow, United Kingdom.

Nogay, H. S. (2017). Deep Convolutional Neural Networks To Detect Lung Cancer Stage, The Journal of Cognitive Systems, 2: 33-36.

Nogay, H. S., Akıncı, T. Ç., Erdemir, G. (2018a). A Convolutional Neural Network Application For The Classification Of Lung Cancer Types. Academic Journal Industrial Technologies, $5: 7-12$.

Nogay, H. S., Akıncı, T. Ç., Erdemir, G. (2018b). Estimation Of Head \& Neck Cancer Stage By Using Deep Convolutional Neural Networks. Academic Journal Industrial Technologies, 5: 13-19.

Nogay, H. S. (2020). Prediction of Post-Treatment Survival Expectancy in Head \& Neck Cancers by Machine Learning Methods", The Journal of Cognitive Systems, 5(1): 23-32.

Nogay, H. S. (2018). Classification Of Different Cancer Types By Deep Convolutional Neural Networks. Balcan Journal of Electrical\&Computer Engineering, 5: 56-59.

Nogay, H. S., Akıncı, T. C. (2018). A Convolutional Neural Network Application For Predicting The Locating Of Squamous Cell Carcinoma In The Lung. Balkan Journal of Electrical \& Computer Engineering, 6: 207-210.

Nogay, H. S., Adeli, H. (2020). Machine learning (ML) for the diagnosis of autism spectrum disorder (ASD) using brain imaging, Reviews In The Neurosciences, DOI: 10.1515/revneuro-2020-0043, 1-17.

Nogay, H. S., Akıncı T. Ç. (2020). Classification of operation cases in electric arc welding wachine by using deep convolutional neural networks, Neural Computing \& Applications, https://doi.org/10.1007/s00521-020-05436-y, 1-14.

Nogay, H. S., Akinci, T. C. (2012). Long term wind speed estimation for a randomly selected time interval by using artificial neural networks, Amasra, Turkey", Energy Education Science and Technology Part A-Energy Science and Research, 28:759772.

Nogay H. S., Akinci T. C., Eidukeviciute M. (2012a) Application of artificial neural networks for short term wind speed forecasting in Mardin, Turkey. Journal of Energy in Southern Africa, 23:2-7.

Noğay, H. S., Akıncı, T. Ç. (2019). Application of decision tree methods for wind speed estimation", European Journal of Technique, cilt.9, ss.74-83.

Nogay, H., S. (2016a). Determination Leakage Reactange in Monophase Transformers Using by Cascaded Neural Network", Balcan Journal of Electrical and Electronical Engineering, 4: 89-96.

Nogay, H. S., Akinci T. C., Guseinoviene E. (2012b).Determination of effect of slot form on slot leakage flux at rotating electrical machines by the method of artificial neural networks", Energy Education Science and Technology Part A-Energy Science and Research, 29:451-462, 2012.

Nogay, H. S. (2008). A Neural Network Solution to Design Dual Stator Winding Insulation Level Detector for Three Phase Induction Motors. WSEAS Transactions on Advances in Engineering Education, 10s.234-240.

Nogay, H. S. (2011). Prediction of internal temperature in stator winding of three-phase induction motors with ann", European Transactions on Electrical Power, 21:120-128.
Nogay, H. S. (2016b). Asenkron Motorda Yapay Sinir Ağları ile Durum Kestirimi. Electronic Journal of Vocational Colleges, $6: 41-48$.

Pinto, G., Ferreira, C., Souza, C., Steinmacher, I., Meirelles, P. (2019). Training Software Engineers sing Open-Source Software: The Students' Perspective. IEEE/ACM 41st International Conference on Software Engineering: Software Engineering Education and Training (ICSE-SEET), 25-31 May, Montreal, QC, Canada.

Pinto, G., Ferreira, C., Souza, C., Steinmacher, I., Meirelles, P. (2017). Training Software Engineers sing Open-Source Software: The Professors' Perspective. The 30th IEEE Conference on Software Engineering Education and Training, 7-9 Nov., Savannah, GA, USA.

Seker, S. S., Akinci, T. C., Nogay, H. S. (2013). Forecasting of wind speed and directions in Kirklareli, Turkey by simple multilayer perceptron, International Symposium on Sustainable Development (ISSD2013), Saraybosna, BosnaHersek, 13 Kasım.

Serteller, N.F.O., Bektas Y., Nogay, S., Akinci, T.C. (2012). Speed Estimation of Brushless Direct Current (BLDC) Motor with Multilayer Perceptron. Przegland Elektrotechniczny, 88:255260.

Suen, H.Y., Hung, K.E., LIN, C.L. (2019). TensorFlow-Based Automatic Personality Recognition Used in Asynchronous Video Interviews. IEEE Access, DOI: 10.1109/ACCESS.2019.2902863.

Tang, L., Mao, X., Zhang, Z. (2019). Language to Code with Open Source Software. IEEE 10th International Conference on Software Engineering and Service Science (ICSESS), 18-20 Oct. Beijing, China.

Tao, Y., Nandigam, J. (2006). Work in Progress: Open Source Software as the Basis of Developing Software Design Case Studies. Proceedings. Frontiers in Education. 36th Annual Conference, 27-31 Oct. San Diego, CA, USA.

Thar, K., Tran, N.H., Oo. T. Z., Hong, C. S. (2018). DeepMEC: Mobile Edge Caching Using Deep Learning, IEEE Access, (6): 78260-78275.

Xie, Y., Qian, K., He, J. (2016). Multi-dimensional and Customizable Open-Source Labware for Promoting Big Data Analytical Skills in STEM Education. 2016 IEEE Frontiers in Education Conference (FIE), 12-15 Oct. Erie, PA, USA.

Volkovas, V., Eidukeviciute, M., Nogay, H. S. , Akinci, T. C. (2012). Application of wavelet transform to defect detection of building's structure, Mechanika, ss.683-690.

Yun, J., Woo, J. (2020). A Comparative Analysis of Deep Learning and Machine Learning on Detecting Movement Directions Using PIR Sensors. IEEE Internet of Things Journal, 7(4):2855-2868. 